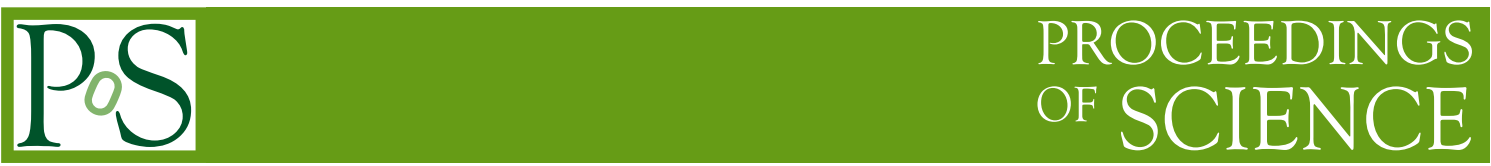

\title{
HI Surveys with APERTIF
}

\author{
Marc Verheijen* \\ University of Groningen, Kapteyn Astronomical Institute \\ Postbus 800, 9700 AV Groningen, The Netherlands \\ E-mail: verheyen@astro.rug.nl
}

\section{Tom Oosterloo, George Heald, Wim van Cappellen}

Netherlands Institute for Radio Astronomy - ASTRON

Postbus 2, 7990 AA Dwingeloo, The Netherlands

E-mail: oosterloodastron.nl

\begin{abstract}
Apertif, the innovative new Focal Plane Array for the Westerbork Synthesis Radio Telescope (WSRT), will significantly increase the instantaneous field-of-view and bandwidth of the WSRT between 1.0 and $1.75 \mathrm{GHz}$. Installing Apertif will tranform the WSRT into an effective survey telescope, enabling efficient northern-sky HI surveys as well as searches for pulsars and transients. In this contribution, we will describe a few strawman HI surveys to illustrate the possibilities. A shallow survey of the northern sky at $\delta>30^{\circ}$ will yield some $10^{5} 8 \sigma$ detections. A similar number of detections can be expected from a medium-deep survey of part of the SLOAN area, reaching lower HI masses and lower column densities to study the extended HI disks and filaments. A deep survey of the Lockman Hole could yield $10^{4}$ detections out to a reshift of $z=0.4$, probing the cosmic evolution of the gas content of galaxies in various environments.
\end{abstract}

Panoramic Radio Astronomy: Wide-field 1-2 GHz research on galaxy evolution - PRA2009

June 02 - 052009

Groningen, the Netherlands

${ }^{*}$ Speaker. 


\section{Introduction}

After more than 40 years, the WSRT will be transformed from one the world's most sensitive and frequency-agile radio telescopes into an efficient survey telescope in the 1.0-1.75 GHz range. The Apertif Focal Plane Array of Vivaldi receivers will enlarge the Field-of-View, within halfpower of the primary beam, from 0.28 to $8.0 \mathrm{deg}^{2}$, the bandwidth from 160 to $300 \mathrm{MHz}$, and the aperture efficiency from 0.55 to 0.70 . The system temperature, however, will increase from 35 to $55 \mathrm{~K}$, such that $\mathrm{A}_{\text {eff }} / \mathrm{T}_{\text {sys }}$ will only decrease by $\sim 20 \%$. Considering the increased bandwidth, the instantaneous sensitivity for continuum sources will actually increase by $\sim 10 \%$. A more elaborate description of Apertif and its prototype is given by Oosterloo et al (this volume).

In general, surveys carried out with Apertif will take advantage of the WSRT being a regular East-West array. For instance, the socalled '8gr8' observing mode exploits the fact that the WSRT behaves like a regular diffraction grating which allows its primary beam to be covered by a set of repeatative tied-array fan-beams that feed into a pulsar machine. Using earth rotation to rotate the fan-beams allows for efficient pulsar searches, yielding accurate locations. Another advantage is that Fourier transforms of the visibilities do not have to take the w-term into account which improves calibration and speeds up the data processing. A draw-back of the WSRT is the fact that it lacks short baselines while the East-West configuration does not allow for acceptable image quality near the celestial equator. Consequently, Apertif will not excell in, for instance, surveys of the Galactic Plane with extended emission or imaging surveys of the sky at low declination.

These considerations make it rather obvious which kind of surveys Apertif will focus on: pulsar surveys, HI imaging surveys of the northern sky at declinations above 30 degrees, and surveys of transients by operating the WSRT in 'Fly's-Eye' mode, pointing each dish at a different location on the sky. Furthermore, to increase the observing efficiency, surveys will be performed commensural as much as possible. For instance, any HI survey will be done in full-polarisation mode with the full $300 \mathrm{MHz}$ bandwidth to allow for polarisation measurements of continuum sources and the determination of rotation measures over the full bandwidth.

It should be noted that the SKA precursors ASKAP and MeerKAT will also engage in HI surveys. The scientific motivations for such surveys is very similar for all three facilities. In order to maximize the overall scientific return, capitalizing on the particular strength of each facility, these surveys should be coordinated. ASKAP will provide the widest instantaneous field-of-view but has the lowest instantaneous sensitivity for line emission. The single pixel feeds of MeerKAT result in a narrow field-of-view but their low system temperatures and the large collecting area turn MeerKAT into the most sensitive array. Apertif holds the middle ground with both a field-of-view and an instantaneous sensitivity in between that of ASKAP and MeerKAT. Furthermore, Apertif is uniquely located in the northern hemisphere allowing for synergetic science with LOFAR.

While allowing for some 'open access time' on the WSRT once equipped with Apertif, it is expected that $\sim 500 \times 12^{\text {hr }}$ per year will be available for surveys for a total period of 5 years. The maximum amount of observing time available for a particular survey will probably not exceed $\sim 200 \times 12^{\mathrm{hr}}$ per year. At the moment, the characteristics of the HI surveys with Apertif are not yet clear and the scientific merits and trade-offs between survey area and survey depth still have to be formulated and evaluated by an international science team. In this contribution, a few strawman surveys will be discussed to illustrate the possibilities. 
Table 1: Several comparative properties of three strawman HI surveys.

\begin{tabular}{l|ccc} 
& shallow & medium & deep \\
\hline Area & northern-sky & SDSS & Lockman Hole \\
& $\delta>+30^{\circ}$ & $8^{\mathrm{hr}}<\alpha<16^{\mathrm{hr}}$ & $\alpha=10 \mathrm{hr} 52 \mathrm{~m}: 43 \mathrm{~s}$ \\
& & $40^{\circ}<\delta<60^{\circ}$ & $\delta=+57^{\circ} 28^{\prime} 48^{\prime \prime}$ \\
Frequency coverage $(\mathrm{MHz})$ & $1430-1130$ & $1430-1130$ & $1300-1000$ \\
Redshift range & $0<\mathrm{z}<0.25$ & $0<\mathrm{z}<0.25$ & $0.09<\mathrm{z}<0.42$ \\
Sky coverage $\left(\mathrm{deg}^{2}\right)$ & 10,300 & 1,500 & 56 \\
number of APERTIF pointings & 1,300 & 192 & 7 \\
$\mathrm{~T}_{\text {int }}$ per pointing & $4^{\mathrm{hr}}$ & $4 \times 12^{\mathrm{hr}}$ & $40 \times 12^{\mathrm{hr}}$ \\
number of $12^{\mathrm{hr}}$ tracks per year & 100 & 156 & 56 \\
rms $($ after Hanning smoothing) $(\mathrm{mJy} / \mathrm{bm})$ & 1.3 & 0.37 & 0.12 \\
number of $8 \sigma$ detections & 105,000 & 92,000 & 8,000 \\
$\mathrm{Z}_{\mathrm{max}}$ for $\mathrm{M}_{\mathrm{HI}}^{*}$ & 0.053 & 0.096 & 0.164 \\
$\mathrm{~N}_{\mathrm{HI}}^{\mathrm{min}}\left(3 \sigma\right.$ for $\Delta \mathrm{v}=50 \mathrm{~km} / \mathrm{s}$ at $\left.\delta=50^{\circ}\right)$ & $5.0 \times 10^{20}$ & $1.4 \times 10^{20}$ & $4.6 \times 10^{19}$ \\
\hline
\end{tabular}

In order to calculate the expected number of detections for a particular survey, we assume a $20 \%$ higher noise than the current WSRT and a $8 \sigma$ detection threshold after optimal spectral smoothing, matching the width of the global profile. For a particular HI mass, a certain line width is adopted following the rough correlation between $\log (\mathrm{W})$ and $\log \left(\mathrm{M}_{\mathrm{HI}}\right)$ from he HIPASS survey (Koribalski et al. 2004); $\log (\mathrm{W})=0.43 \log (\mathrm{MHI})-1.72$. The relatively high significance is warranted for a blind survey which is largely dominated by noise. Furthermore, it is assumed that the galaxies are spatially unresolved. Lower detection thresholds can be accepted in case optical counterparts exist or optical redshifts are available.

\section{A shallow northern-sky survey}

Maximizing the number of HI detections in the trade-off between survey area and survey depth for a fixed amount of time, favours the largest possible sky coverage. In such a wide-area survey, the population of HI detected galaxies will be dominated by relatively nearby, gas-rich and lowmass galaxies. Since most detections will be in the nearby universe, the $300 \mathrm{MHz}$ bandwidth of Apertif should start at $1430 \mathrm{MHz}$ to include galaxies with negative recession velocities, and reach down to $1130 \mathrm{MHz}$ which corresponds to $\mathrm{z}=0.25$.

ASKAP will perform a shallow southern-sky HI survey, called Wallaby, up to a declination of +30 degrees (Staveley-Smith, this volume). To increase the legacy value of the Apertif and ASKAP shallow surveys, the survey characteristics should be properly matched in terms of complementary sky coverage and a similar redshift range, spectral resolution and sensitivity. To obtain a truly 'all-sky' survey, Apertif should survey the entire northern sky above a declination of $\delta>+30^{\circ}$, comprising 10,300 $\mathrm{deg}^{2}$. This can be covered with 1,300 Apertif pointings. To obtain the same sensitivity as the Wallaby survey, an integration time with Apertif of 4 hours per pointing is required. Allowing for calibration and slewing overheads, a total of 6,000 hours would be required. With a survey period of 5 years, this amounts to $100 \times 12^{\text {hr }}$ per year. 


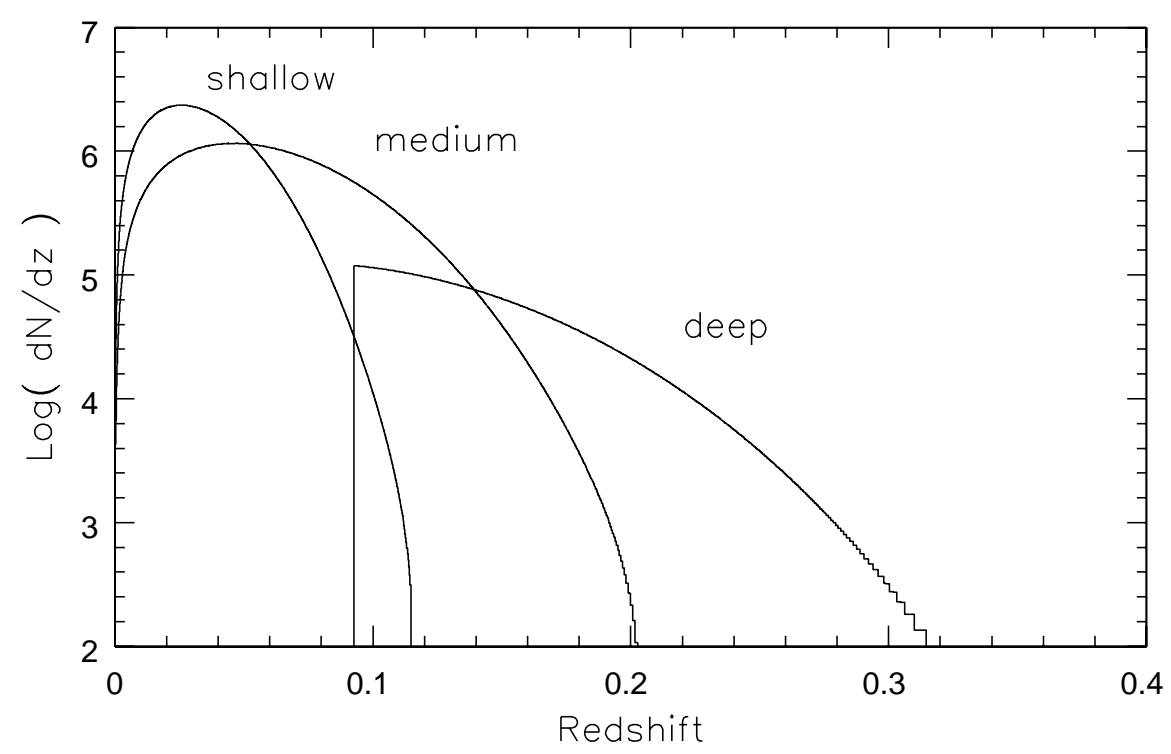

Figure 1: Redshift distributions for the three strawman surveys listed in Table 1. The normalisations are based on the total number of expected detections.

With an integration time of only 4 hours per Apertif pointing, a galaxy with a typical HI mass of $\mathrm{M}_{\mathrm{HI}}^{*}=10^{9.8} \mathrm{M}_{\odot}$ can be detected at the $8 \sigma$-level out to $\mathrm{z}=0.053$, assuming optimal spectral smoothing to the expected line width. The most gas-rich galaxies with $\mathrm{HI}$ masses of $\mathrm{M}_{\mathrm{HI}}=5 \times 10^{10}$ $\mathrm{M}_{\odot}$ can be detected at the same significance level out to $\mathrm{z}=0.11$. The total number of $8 \sigma$ detections is expected to be $1 \times 10^{5}$, assuming that the galaxies are spatially unresolved which is obviously not the case. On the other hand, the HI mass - line width relation traces the upper envelope of the line width distribution and is thus representative for galaxies close to an edge-on orientation. Less inclined galaxies will have narrower line widths and will be easier to detect.

The column density sensitivity, which is roughly distance independent for this redshift range, will be rather limited due to the relatively short integration time per pointing. The $3 \sigma$ detection limit for column density will be $\mathrm{N}_{\mathrm{HI}}=5 \times 10^{20}$ atoms $/ \mathrm{cm}^{2}$ for a profile width of $50 \mathrm{~km} / \mathrm{s}$ and a synthesized beam of $15^{\prime \prime} \times 15^{\prime \prime} / \sin \left(50^{\circ}\right)$.

The shallowness of the combined Apertif/ASKAP survey implies that almost no individual galaxies will be detected in the redshift range $0.11<z<0.25$. Nevertheless, observing with the full $300 \mathrm{MHz}$ bandwidth should be considered to maximize sensitivity for radio continuum sources. It also allows for statistical HI detections by stacking the HI spectra using optical redshifts.

A shallow all-sky HI survey will provide a measurement of, or an upper limit for, the gas content of every single galaxy in the nearby universe. This allows the study of the gas content of nearby galaxies as a function of luminosity, colour, morphology, star formation rate, environment, etc. Furthermore, the width of the global HI profiles will provide the dynamical masses of galaxies and can be used for Tully-Fisher studies, sampling the entire local volume of the universe. Furthermore, it will map the detailed structure of High Velocity Clouds surrounding the Milky Way, covering the entire sky. 


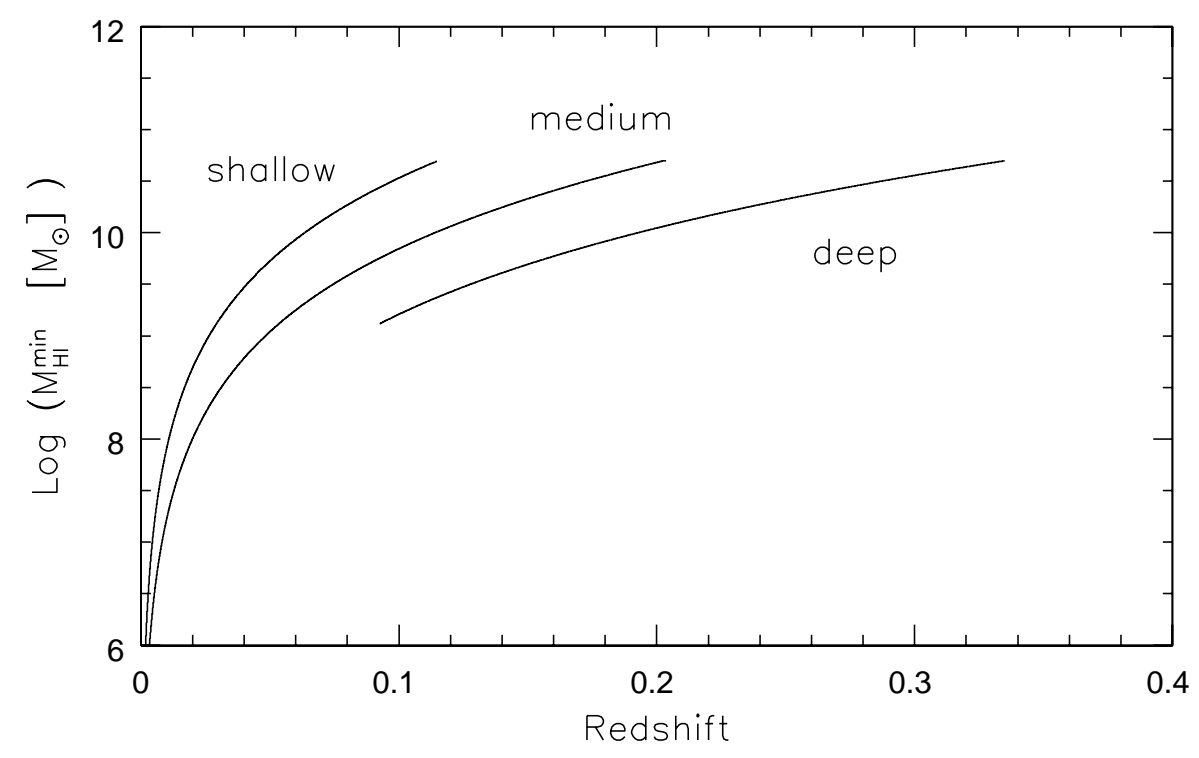

Figure 2: Minimum detectable $\mathrm{HI}$ mass as a function of redshift for the three strawman surveys specified in Table 1. A detection limit of $8 \sigma$ after optimal spectral smoothing and a maximum HI mass of $5 \times 10^{10} \mathrm{M}_{\odot}$ are assumed.

\section{A medium-deep survey}

The main limitation of a shallow survey is the fact that galaxies with a low HI mass can only be detected in case they are very nearby, making it difficult to obtain accurate distances based on their recession velocities. Furthermore, the limited column density sensitivity of a shallow survey prohibits the detection of extended diffuse gas around galaxies.

The main motivation for a medium-deep survey is to study the detailed morphologies and kinematics of the HI gas in galaxies. On the one hand, this requires good column density sensitivity to map warps, tidal tails and other filamentary structure in the outer regions of galaxies. On the other hand, a wide range of global and local environments, ranging from voids to the cores of galaxy clusters, need to be sampled extensively to study the influence of the environment on the efficiency of physical mechanisms that play a role in the accretion and depletion of gas in galaxies. Obtaining good angular resolution means that the galaxies can not be too distant. Ensuring a good sampling of many different environments means that a relatively large volume of the local universe should be observed. The extragalactic sky area covered by the SLOAN survey offers the best opportunity for a medium-deep survey. The area of $8^{\mathrm{hr}}<\alpha<16^{\mathrm{hr}}$ and $40^{\circ}<\delta<60^{\circ}$ spans $1,535 \mathrm{deg}^{2}$ which can be covered by 192 Apertif pointings.

In order to reach a column density sensitvity of $\mathrm{N}_{\mathrm{HI}}=10^{19}$ atoms $/ \mathrm{cm}^{2}$ at $3 \sigma$ over a profile width of $50 \mathrm{~km} / \mathrm{s}$ at an angular resolution of $1 \mathrm{arcmin}$, a total intergration time of $4 \times 12^{\mathrm{hr}}$ is required. This column density and angular resolution are sufficient to image extended HI filaments like those found in the Virgo cluster due to ram-pressure stripping (Oosterloo \& van Gorkom, 2005) and in the Ursa Major cluster due to tidal interactions. Investing $4 \times 12^{\mathrm{hr}}$ of integration time per pointing would thus require $156 \times 12^{\mathrm{hr}}$ per year for a period of 5 years. 
In such a medium-deep survey, an $\mathrm{HI}$ mass of $10^{5} \mathrm{M}_{\odot}$ can be detected at $8 \sigma$ from a distance of $4.5 \mathrm{Mpc}$, while $\mathrm{M}_{\mathrm{HI}}^{*}$ can be detected at a redshift of 0.096 . The most gas-rich galaxies with a mass of $5 \times 10^{10} \mathrm{M}_{\odot}$ can still be detected at $\mathrm{z}=0.2$. The total number of detected galaxies in such a medium-deep survey will be $\sim 9 \times 10^{4}$, sufficient for studies of multi-variate dependencies of the gas properties like the occurence of warps as a function of morphological type, dynamical mass and local environment.

\section{A deep survey}

A medium-deep survey as sketched above will not be able to detect even the most gas-rich galaxies beyond a redshift of 0.2 while the low-frequency limit of the Apertif system can reach down to $1000 \mathrm{MHz}$ or $\mathrm{z}=0.4$, with the $300 \mathrm{MHz}$ bandwidth instanteneously covering the redshift range from 0.09 to 0.4 . To properly study the gas properties of galaxies at these cosmologically significant distances, more sensitivity is required. In order to detect, at the $8 \sigma$-level, $10^{9} \mathrm{M}_{\odot}$ at $\mathrm{z}=0.1,10^{10} \mathrm{M}_{\odot}$ at $\mathrm{z}=0.2$, and $5 \times 10^{10} \mathrm{M}_{\odot}$ at $\mathrm{z}=0.35$, an integration time of $40 \times 12^{\mathrm{hr}}$ is required. This allows, at the same significance level, a galaxy with $\mathrm{M}_{\mathrm{HI}}^{*}=10^{9.8} \mathrm{M}_{\odot}$ to be detected at $\mathrm{z}=0.17$ as indicated in Figure 2.

The Lockman Hole is an obvious region of the sky to be selected for a deep survey. This area of minimal Galactic extinction near the North Galactic Pole has been surveyed with many different facilities at many different wavelengths. Assigning 7 Apertif pointings, comprising 56 square degrees, fully covers the Lockman Hole and the distant volume surveyed with Apertif is expected to contain $\sim 8 \times 10^{3}$ detectable galaxies.

Within the highest redshift range that can be observed with Apertif, signs of cosmological evolution are becoming evident. The nearest Butcher-Oemler clusters are found at these redshifts and it is expected that the atomic gas density $\Omega_{\mathrm{HI}}$ of the universe is enhanced with respect to $\mathrm{z}=0$. A deep survey with Apertif, resulting in nearly $10^{4} \mathrm{HI}$ detected galaxies at redshifts 0.1 to 0.4 will be able to reveal the cosmological evolution of the gas content of galaxies in different environments.

\section{Conclusion}

Of course, the three strawman surveys outlined above and summarized in Table 1 merely illustrate the possibilities. The details of actual HI surveys to be carried out with Apertif in terms of sky coverage, depth and selected areas, will be discussed and finalized within an international science team. It is noteworthy, however, that carrying out all 3 strawman surveys sketched above requires a total observing time of $300 \times 12^{\mathrm{hr}}$ per year for a period of 5 years which leaves some $200 \times 12^{\mathrm{hr}}$ per year available for pulsar and transient surveys.

\section{References}

[1] Koribalski, B.S., Staveley-Smith, L. Kilborn, V.A. et al 2004, A.J., 128, 16

[2] Oosterloo, T.A. and van Gorkom, J.H. 2005, A.\& A., 437, L19 